\title{
Temperature and Strain Sensing With Femtosecond Laser Written Bragg Gratings in Defect and Nondefect Suspended-Silica-Core Fibers
}

\author{
Luís André Fernandes, Martin Becker, Orlando Frazão, Kay Schuster, Jens Kobelke, Manfred Rothhardt, \\ Hartmut Bartelt, José Luís Santos, and Paulo V. S. Marques
}

\begin{abstract}
The spectral behavior in the C-band of fiber Bragg gratings (FBGs) was analyzed as a function of temperature and strain. The FBGs were fabricated in pure silica four-leafclover-shaped suspended-core fibers by (DUV) femtosecond laser exposure $(3.6 \mathrm{~W}$ at $800 \mathrm{~nm}, 130 \mathrm{fs}, 1 \mathrm{kHz}$ frequency tripled to $350 \mathrm{fs}, 650 \mathrm{~mW}$ at $267 \mathrm{~nm}$ ). A defect fiber (with a hollow hole in the core) and nondefect fiber were compared both yielding $\approx 1 \mathrm{pm} / \mu \epsilon$ sensitivity to strain but different sensitivity to temperature (from $3.0 \mathrm{pm} /{ }^{\circ} \mathrm{C}$ to $8.4 \mathrm{pm} /{ }^{\circ} \mathrm{C}$ for the defect fiber and $10 \mathrm{pm} /{ }^{\circ} \mathrm{C}$ for the nondefect fiber). The $16 \%$ to $70 \%$ relative difference between the thermal coefficients of the two fibers, together with their similar strain sensitivity enables the simultaneous measurement of strain and temperature.
\end{abstract}

Index Terms-Femtosecond laser, fiber Bragg gratings (FBGs), suspended core optical fibers temperature and strain measurement, temperature sensitivity, strain sensitivity.

\section{INTRODUCTION}

$\mathbf{F}$ IBER Bragg gratings (FBGs) have been extensively used in telecommunication and sensing applications, particularly in strain and temperature sensing due to the attractiveness of the fiber substrate to be used for distributed sensing or under hazardous environments. Besides the standard single mode fibers commonly used, other fibers have been found to be particularly useful for some sensing application like photonic crystal fibers (PCFs) [1] or suspended core fibers (SCFs) [2]. The geometry of an SCF makes them specially attractive for evanescent field sensing [3] and fluorescence spectroscopy [4], however, due to the absence of a doped core and other difficulties found with hydrogen loading, conventional UV laser writing techniques are not effective in the fabrication of FBGs [5]. The use of femtosecond laser pulses [6] opened the possibility for creating refractive index modulations in the pure silica core, due to the nonlinear absorption observed when the

Manuscript received October 5, 2011; revised December 12, 2011; accepted January 3, 2012. Date of publication January 9, 2012; date of current version March 7, 2012.

L. A. Fernandes, O. Frazão, J. L. Santos, and P. V. S. Marques are with the Departamento de Física e Astronomia da Universidade do Porto, INESC-Porto, Porto 4169-007, Portugal (e-mail: lfernandes@ fc.up.pt; ofrazao@inescporto.pt; josantos@fc.up.pt; psmarque@fc.up.pt).

M. Becker, K. Schuster, J. Kobelke, M. Rothhardt, and H. Bartelt are with the Institute of Photonic Technology, Jena D-07745, Germany (e-mail: martin.becker@ipht-jena.de; kay.schuster@ipht-jena.de; jens.kobelke@iphtjena.de; manfred.rothhardt@ipht-jena.de; hartmut.bartelt@ipht-jena.de).

Color versions of one or more of the figures in this letter are available online at http://ieeexplore.ieee.org.

Digital Object Identifier 10.1109/LPT.2012.2183344 high peak intensity of a femtosecond laser pulse is focused in the transparent core. The SCFs used in this letter were already tested as an interferometric sensor [7].

In this letter, we use a femtosecond laser writing system developed by IPHT [5] and two SCFs to demonstrate simultaneous temperature and strain measurements. The main advantage provided by these fibers is the possibility of controlling the temperature sensitivity using different core geometries. However, this approach is limited by the difficulty in splicing SCFs with single mode fibers (SMFs).

\section{FABRICATION}

The fabrication system is described in [5] and consists of a femtosecond infrared oscillator pulse (Coherent Mantis) and a regenerative Ti:Sapphire amplifier (Coherent Legend Elite) which produces $130 \mathrm{fs}$ pulses with $800 \mathrm{~nm}$ center wavelength, $3.6 \mathrm{~W}$ average power at $1 \mathrm{kHz}$ repetition rate. A third harmonic generation unit produces $\approx 350$ fs pulses with $650 \mathrm{~mW}$ average power centered at $267 \mathrm{~nm}$. To define the Bragg grating structures, the beam is focused through a $335 \mathrm{~nm}$ focal length cylindrical lens into a Talbot interferometer composed by a phase mask (1065.3 $\mathrm{nm}$ periodicity), that splits the beam, and two computer controlled rotation mirrors. The two first diffraction orders from the phase mask are reflected by the mirrors and interfered in the focal plane of the cylindrical lens, where the fiber is positioned. The angle of the mirrors together with the phase mask period define the periodicity of the interference pattern as described in [8], [9].

The fibers used, custom made by IPHT, are pure silica suspended core fibers that consist of a silica core, connected by thin silica bridges to the the fiber cladding. The hollow holes around the core provide the confinement needed for single mode waveguide operation in the C-band. A facet view of the fibers used in this demonstration is shown in Fig. 1. The IPHT-256b5 (Fig. 1b) has a $7.2 \mu \mathrm{m}$ diameter core and $123 \mu \mathrm{m}$ diameter cladding while the IPHT-256b2 (Fig. 1a) has a $4.9 \mu \mathrm{m}$ diameter core, a defect hollow hole inside the core with $1.4 \mu \mathrm{m}$ diameter and a $106 \mu \mathrm{m}$ diameter cladding. This hollow hole is considered a defect when its dimensions are similar to the wavelength and therefore small enough not to affect the single mode propagation while significantly contributing to the effective index of the waveguide. To measure the reflected spectrum during fabrication, the SCFs were spliced with standard SMFs and the process was optimized to reduced 

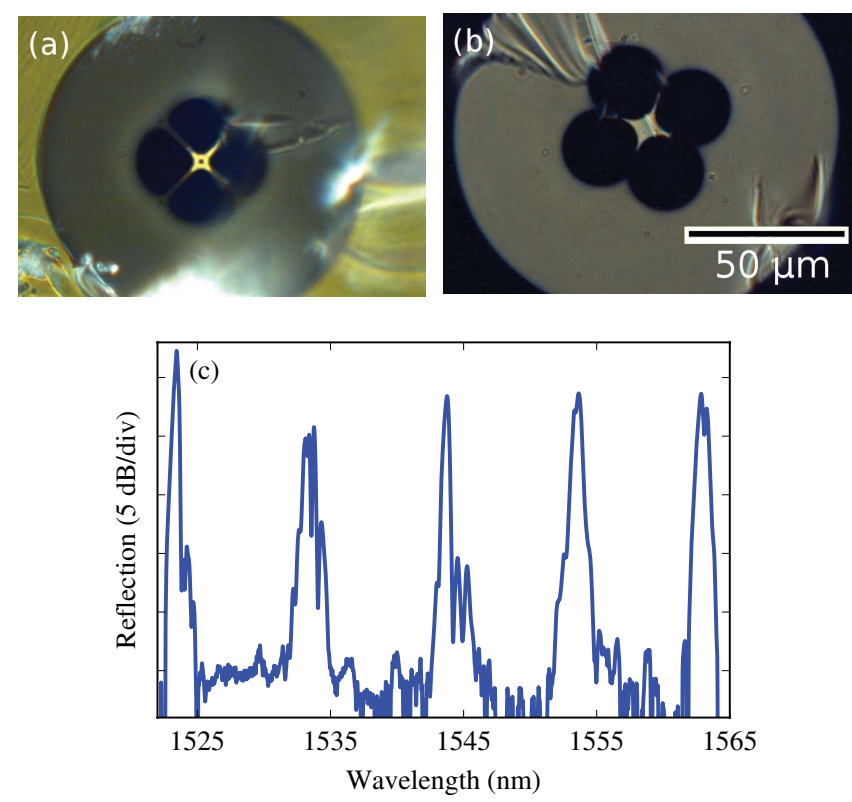

Fig. 1. Microscope images of the facets of two pure silica suspended core fibers: (a) defect IPHT-256b2 and (b) nondefect IPHT-256b5. (c) Reflection spectrum of an array of FBGs writen in the IPHT-256b5 fiber.

reflections and prevent interference with the FBGs signal. The propagation losses in the SCFs is in the order of tens of $\mathrm{dB} / \mathrm{km}$.

\section{THEORETICAL ANALYSIS}

The first derivative of the Bragg relation, $\lambda_{B}=2 n \Lambda$, with respect to temperature $T$ is given by Equation 1 .

$$
\frac{d \lambda_{B}}{d T}=2 \frac{d \Lambda}{d T} n+2 \Lambda \frac{d n}{d T}
$$

Where $\lambda_{B}$ is the Bragg resonance, $n$ is the effective index of the waveguide mode and $\Lambda$ is the periodicity of the FBG. $d \lambda_{B} / d T$ is the temperature sensitivity of the FBG and $d \Lambda / d T=\Lambda \alpha_{L}$, where $\alpha_{L}$ is the linear thermal expansion coefficient and $d n / d T$ is the thermo optic effect in the waveguide mode. A similar analysis can be found in [10] for evanescent field sensing.

The simplification of Equation 1 by taking into account the thermal expansion coefficient and the Bragg relation yields:

$$
\frac{d \lambda_{B}}{d T}=\lambda_{B} \alpha_{L}+\frac{\lambda_{B}}{n} \frac{d n}{d T}
$$

In Equation 2 it is clear that the temperature variation has two effects over the Bragg wavelength shift: The thermal expansion coefficient of the FBG is considered in the first term, increasing the reflected wavelength with temperature, while the second term reflects the thermo optic effect, which changes the refractive index of the waveguide mode with temperature. For pure silica, the thermal expansion coefficient, $\alpha_{L}$, is $5.5 \times 10^{-7} \mathrm{~K}^{-1}$ [11] and the thermo optic coefficient is $8.6 \times 10^{-6} \mathrm{~K}^{-1}$ at $300 \mathrm{~K}$ and $1.5 \mu \mathrm{m}$ wavelength [12]. For air, the thermo optic coefficient is $-9 \times 10^{-7} \mathrm{~K}^{-1}$ [13].

In Equation 2, the presence of air in the defect located in the center of the core of the IPHT-256b2 fiber will reduce the overall thermo optic coefficient of the guided mode, therefore reducing the temperature sensitivity of the FBG when
TABLE I

Material Properties

\begin{tabular}{|c|c|c|}
\hline Material & $\begin{array}{c}\text { Linear thermal expansion } \\
\text { coefficient, } \alpha_{L}\end{array}$ & $\begin{array}{c}\text { Thermo optic } \\
\text { coefficient, } d n / d T\end{array}$ \\
\hline \hline Fused silica & $5.5 \times 10^{-7} \mathrm{~K}^{-1}$ & $8.6 \times 10^{-6} \mathrm{~K}^{-1}$ \\
Air & - & $-9 \times 10^{-7} \mathrm{~K}^{-1}$ \\
IPA & - & $-3.4 \times 10^{-4} \mathrm{~K}^{-1}$ \\
\hline
\end{tabular}

compared to the IPHT-256b5 fiber. Other materials like water (with a thermo optic coefficient of $-8 \times 10^{-5} \mathrm{~K}^{-1}$ [14]) or isopropyl alcohol (IPA) (with a thermo optic coefficient of $\left.-3.4 \times 10^{-4} \mathrm{~K}^{-1}[15]\right)$ will have an even higher effect in reducing the FBG's temperature sensitivity. Table I summarizes the material properties relevant to this letter.

The presence of the defect in the IPHT-256b2 fiber will then have a significant contribution to the temperature sensitivity of the written FBG since it will change the thermo optic coefficient of the waveguide mode. This can be useful to tune the sensitivity and perform simultaneous temperature and strain sensing, as well as refractive index sensing with higher sensitivity than that obtained by evanescent field based measurements.

\section{RESULTS}

The strain characterization of the FBG fabricated was performed by carefully attaching the fibers to a translation stage with $1 \mu \mathrm{m}$ resolution that is used to apply tension to the grating region. The grating response to temperature is studied using a tubular oven with an error smaller than $0.1{ }^{\circ} \mathrm{C}$.

Figure 2 shows the Bragg resonance wavelength and the Bragg resonance wavelength variation respectively as a function of strain (Fig. 2a) and temperature (Fig. 2b) for both IPHT-256b2 and IPHT-256b5 suspended core fibers. The strain dependence measured in Fig. 2a shows that both fibers have very similar behavior with strain, yielding a sensitivity, $d \lambda_{B} / d \epsilon$, of $(1.15 \pm 0.01) \mathrm{pm} / \mu \epsilon$ and $(1.14 \pm 0.01) \mathrm{pm} / \mu \epsilon$ for the IPHT-256b2 and IPHT-256b5 fibers, respectively. The thermal sensitivity is clearly shown to be different in Fig. 2b where the Bragg resonance wavelength variation is plotted as a function of the temperature.

Sensitivities of $(8.42 \pm 0.05) \mathrm{pm} /{ }^{\circ} \mathrm{C}$ for the IPHT-256b2 fiber with air filling the defect and $(10.0 \pm 0.2) \mathrm{pm} /{ }^{\circ} \mathrm{C}$ for the IPHT$256 \mathrm{~b} 5$ fiber were found. If IPA is used in the $256 \mathrm{~b} 2$ fiber defect instead of air, the sensitivity to temperature further decreases to $(3.0 \pm 0.1) \mathrm{pm} /{ }^{\circ} \mathrm{C}$ with the IPA filling not only the central air hole but also the four lateral air holes in the SCF.

With the physical constants summarized in Table I for fused silica and using Equation 2, an approximate theoretical value of $10 \mathrm{pm} /{ }^{\circ} \mathrm{C}$ was found for the temperature sensitivity of the nondefect IPHT-256b5 fiber, assuming that $d n / d T$ is approximately the same in the waveguide as in bulk silica. This result agrees well with the $10.0 \pm 0.2 \mathrm{pm} /{ }^{\circ} \mathrm{C}$ experimental result. The $16 \%$ to $70 \%$ temperature sensitivity variation between the two fibers may be accounted for by the difference in the thermo optic coefficient between fused silica, air and IPA.

Using the sensitivity results from Table II, it is possible to calculate the solution for a measurement of unknown temper- 

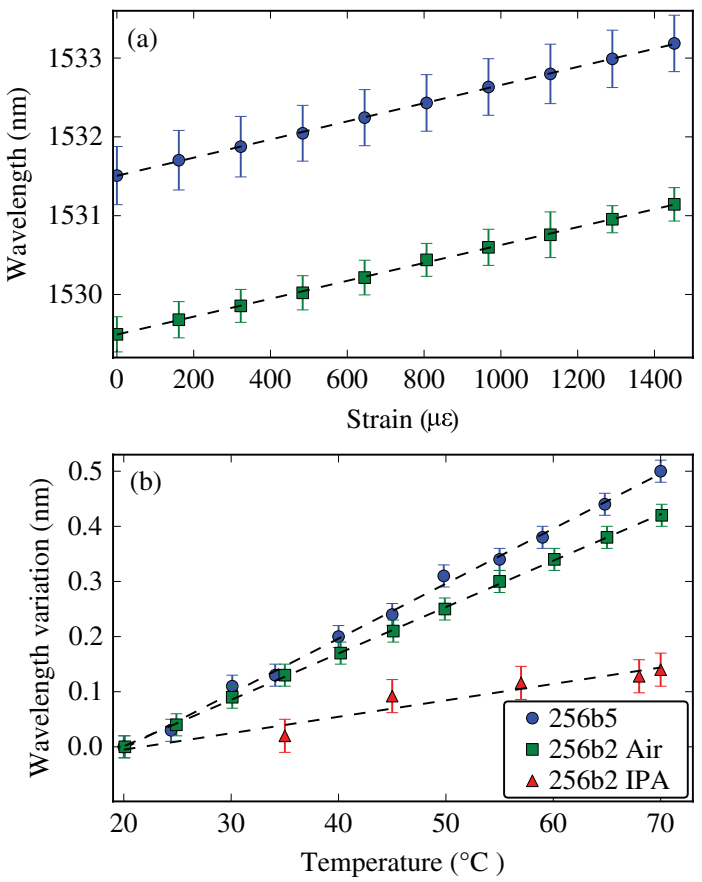

Fig. 2. (a) Bragg wavelength as a function of applied strain for the two fibers tested. (b) Variation as a function of the temperature of the Bragg wavelength compared to the FBG resonance at the starting temperature of $20{ }^{\circ} \mathrm{C}$. In both graphics, the $(\bullet)$ blue circle represents the experimental data from the IPHT$256 \mathrm{~b} 5$ fiber and the $(\square)$ green square represents the experimental data from the IPHT-256b2 fiber with air in the hollow defect. In (b) the ( $\mathbf{\Delta})$ red triangle represents the experimental data from the IPHT-256b2 fiber with IPA in the hollow defect. The (- -) dashed lines represent the best linear fit of each experimental data set.

TABLE II

SENSITIVITY EXPERIMENTAL RESULTS

\begin{tabular}{|c|c|c|}
\hline Fiber & $\begin{array}{c}\text { Temperature } \\
\text { sensitivity } d \lambda_{B} / d T\end{array}$ & $\begin{array}{c}\text { Strain sensitivity } \\
d \lambda_{B} / d \epsilon\end{array}$ \\
\hline \hline IPHT-256b5 & $10.0 \pm 0.2 \mathrm{pm} /{ }^{\circ} \mathrm{C}$ & $1.14 \pm 0.01 \mathrm{pm} / \mu \epsilon$ \\
IPHT-256b2 (Air) & $8.42 \pm 0.05 \mathrm{pm} /{ }^{\circ} \mathrm{C}$ & $1.15 \pm 0.01 \mathrm{pm} / \mu \epsilon$ \\
IPHT-256b2 (IPA) & $3.0 \pm 0.1 \mathrm{pm} /{ }^{\circ} \mathrm{C}$ & - \\
\hline
\end{tabular}

ature and strain based on Bragg grating shift measurements made in both fibers.

$$
\begin{array}{r}
\Delta \lambda_{b 5}=K_{T_{1}} \Delta T+K_{\epsilon_{1}} \Delta \epsilon \\
\Delta \lambda_{b 2}=K_{T_{2}} \Delta T+K_{\epsilon_{2}} \Delta \epsilon \\
\left|\begin{array}{ll}
K_{T_{1}} & K_{\epsilon_{1}} \\
K_{T_{2}} & K_{\epsilon_{2}}
\end{array}\right|=D \neq 0
\end{array}
$$

Where $K_{\epsilon}$ is the strain sensitivity, $d \lambda_{B} / d \epsilon$, and $K_{T}$ is the temperature sensitivity, $d \lambda_{B} / d T$. The determinant, $D$, of the matrix in Equation 4 is different than zero which proves that a solution to the set of Equations 3 is possible. Using the sensitivity values from Table II, the determinant for the set of equation using the IPHT-256b2 fibers with air in the defect is $D=1.9 \mathrm{pm}^{2} \mu \epsilon^{-1{ }^{\circ}} \mathrm{C}^{-1}$ while using the same fiber with

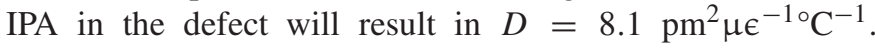
As demonstrated in [16] a higher determinant value, as the one obtained with IPA, allows for higher accuracy in the temperature and strain simultaneous measurement.
The IPHT-256b2 fiber was also characterized as a function of pressure, with a $1 \mathrm{MPa}$ of pressure variation applied, but no measurable shift in the Bragg wavelength was found.

\section{CONClusion}

These results show that the two fibers presented can be used to make simultaneous measurements of temperature and strain. In such a configuration where both fibers are subject to the same amount of applied tension, both would shift approximately the same amount due to that stress while a variation in the difference between the two FBGs would account for the temperature change. The temperature sensitivity dependence in the material filing the defect inside the core of the defect IPHT$256 \mathrm{~b} 2$ fiber may be used to tune the temperature sensitivity of an FBG in such fiber, further increasing the flexibility for sensor design. Furthermore, if temperature sensitivity is tuned to be negligible in a certain range, a strain sensor immune to temperature fluctuations can be demonstrated.

\section{REFERENCES}

[1] J. Knight, T. Birks, P. Russell, and D. Atkin, "All-silica single-mode optical fiber with photonic crystal cladding," Opt. Lett., vol. 21, no. 19, pp. 1547-1549, 1996.

[2] A. Webb, F. Poletti, D. Richardson, and J. Sahu, "Suspended-core holey fiber for evanescent-field sensing," Proc. SPIE: Opt. Eng., vol. 46, p. 010503, Jan. 2007.

[3] M. P. Huy, et al., "Three-hole microstructured optical fiber for efficient fiber Bragg grating refractometer," Opt. Lett., vol. 32, no. 16, pp. 23902392, 2007.

[4] V. S. Afshar, S. Warren-Smith, and T. Monro, "Enhancement of fluorescence-based sensing using microstructured optical fibres," Opt. Express, vol. 15, no. 26, pp. 17891-17901, 2007.

[5] M. Becker, et al., "Inscription of fiber Bragg grating arrays in pure silica suspended core fibers," IEEE Photon. Technol. Lett., vol. 21, no. 19, pp. 1453-1455, Oct. 1, 2009.

[6] K. Davis, K. Miura, N. Sugimoto, and K. Hirao, "Writing waveguides in glass with a femtosecond laser," Opt. Lett., vol. 21, no. 21, pp. 17291731, 1996.

[7] O. Frazao, J. Baptista, J. Santos, J. Kobelke, and K. Schuster, "Strain and temperature characterisation of sensing head based on suspendedcore fibre in Sagnac interferometer," Electron. Lett., vol. 44, no. 25, pp. 1455-1456, Dec. 2008.

[8] M. Becker, et al., "Fiber Bragg grating inscription combining DUV subpicosecond laser pulses and two-beam interferometry," Opt. Express, vol. 16, no. 23, pp. 19169-19178, 2008.

[9] T. Geernaert, et al., "Fiber Bragg gratings in germanium-doped highly birefringent microstructured optical fibers," IEEE Photon. Technol. Lett., vol. 20, no. 8, pp. 554-556, Apr. 15, 2008.

[10] R. Kamikawachi, et al., "Determination of thermo-optic coefficient in liquids with fiber Bragg grating refractometer," Opt. Commun., vol. 281, no. 4, pp. 621-625, 2008.

[11] T. F. Retajczyk and A. K. Sinha, "Elastic stiffness and thermal expansion coefficients of various refractory silicides and silicon nitride films," Thin Solid Films, vol. 70, no. 2, pp. 241-247, 1980.

[12] D. B. Leviton and B. J. Frey, "Temperature-dependent absolute refractive index measurements of synthetic fused silica," Proc. SPIE, vol. 6273, pp. 62732K-1-62732K-11, May 2006.

[13] H. Bach and N. Neuroth, The Properties of Optical Glass. New York: Springer-Verlag, 1995.

[14] A. Chryssis, S. Saini, S. Lee, and M. Dagenais, "Increased sensitivity and parametric discrimination using higher order modes of etched-core fiber Bragg grating sensors," IEEE Photon. Technol. Lett., vol. 18, no. 1, pp. 178-180, Jan. 1, 2006.

[15] C. Kim and C. Su, "Measurement of the refractive index of liquids at 1.3 and 1.5 micron using a fibre optic Fresnel ratio meter," Meas. Sci. Technol., vol. 15, no. 9, pp. 1683-1686, 2004.

[16] W. Jin, W. Michie, G. Thursby, M. Konstantaki, and B. Culshaw, "Simultaneous measurement of strain and temperature: Error analysis," Proc. SPIE: Opt. Eng., vol. 36, no. 2, pp. 598-609, 1997. 